\title{
PCORNet Trial Domain
}

National Cancer Institute

\section{Source}

National Cancer Institute. PCORNet Trial Domain. NCI Thesaurus. Code C154634.

Patients who are enrolled in PCORnet clinical trials and PCORnet studies. 